\title{
QUADCOPTER WITH THRUST VECTOR CONTROL BASED ON SCOTCH YOKE
}

\author{
SERGEY V. SEMENDYAEV \\ Moscow Institute of Physics and Technology (MIPT) \\ 9 Institutskiy per., Dolgoprudny, Moscow Region, 141701, Russian Federation \\ e-mail: semendyaevsergey@gmail.com, web page: https://mipt.ru/english/
}

Key words: Quadcopter, Thrust Vector Control, Scotch Yoke.

\begin{abstract}
People are interested in quadcopters (or quadrotors) because of flight maneuverability and a variety of applications. Standard quadrotor has constant mechanical structure - frame with four motors and four rotors (propellers), and usually, rotors do not change the angle of the rotation axis relative to the frame. However, there are several designs in which the axis of rotation of rotors changes the angle relative to the frame (for example, "Holocopter" and "Tilt-rotor"), and this option changes the thrust vector of the quadcopter. In this work a novel design of thrust vector control based on the Scotch yoke is proposed. All rotor axes incline simultaneously in any direction by only two servos. Construction design is proposed, and general dynamics are described.
\end{abstract}

\section{INTRODUCTION}

In conventional quadcopters, the structure is fixed, and the thrust vector is controlled by changing the speed of rotation of different propellers. But there are designs in which the angle of inclination of the rotor axis changes, for example, as in "Holocopter" (where the propellers axles rotate relative to the axes coaxial to the beams of the quadcopter) [1] and "Tilt-rotor" [29]. In this case, the change in the thrust vector is carried out not only by the speed of rotation of the rotors but also by tilting their axis of rotation.

A quadcopter is an aircraft that has four motors with propellers that create thrust. Typically, the propeller axes and blade angles are fixed, and only rotational speeds are controlled, which greatly simplifies the design. Vertical movement is carried out by a synchronous change in the rotation speeds of all propellers; for horizontal movement, you need to tilt the quadcopter, which is achieved by a corresponding change in the rotation speeds of different propellers that create the moments necessary for tilt. The opposite direction of the pairs of propellers provides compensation for the moments of drag. Such devices with fixed propeller axes are used quite widely.

In this paper, it is proposed to investigate the possibilities of tilting the propeller axes to create a deflected thrust vector and not tilt the quadcopter for horizontal movement. As a result, - to reduce aerodynamic drag, to increase the speed and maneuverability of flight.

Adding variable axle tilt to the propellers results in additional degrees of freedom for varying the thrust generated by each motor-propeller group. With propellers of this type, the thrust can be changed by changing the angle of inclination of the propeller axles in projection 
on the horizontal plane and the vertical. The angles of inclination of the propeller axles and the speed of rotation of the propeller motors - these two factors overlap the range of action, since, for example, for forward motion we can quickly rotate the propellers at the stern, and slow down the speeds of the motors at the bow, and on the other hand we can tilt the axles of propellers for the same purpose. However, in the first case, we have to increase the pitch angle of the quadcopter, and in the second, keeping the pitch angle constant, tilt the propeller axles. The number of possible combinations between the body pitch and pitch of the propellers for forward motion is limited by the maximum pitch angles of the propellers, caused by the maximum engine power, the capabilities of the motion control devices, construction and aerodynamic constraints.

In the quadcopters with a variable angle of inclination of the propeller axles considered in this article, all axles are tilted by the same angle, in the desired direction of movement. So, the quadcopters can quickly accelerate and stop quickly while maintaining the horizontal orientation of the body. The quadcopter can also tilt the body, keeping the barycenter velocity zero, which is impossible in conventional quadcopters.

\section{CONSTRUCTION DESIGN}

\section{There is a standard layout for the quadcopter called X4 (see Fig. 1).}
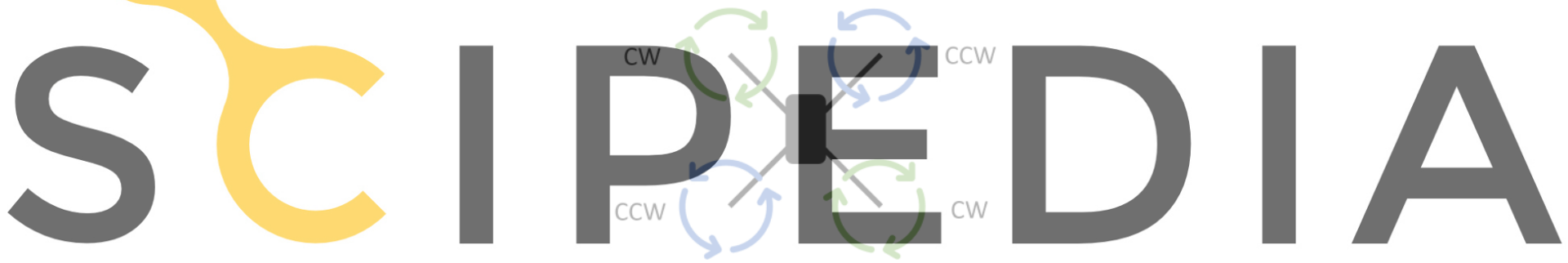

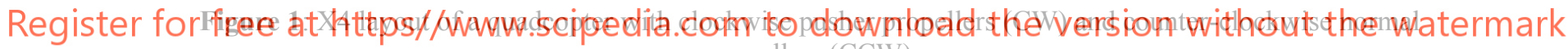
propellers (CCW)

At the moment we will give preference to the $\mathrm{X} 4$ scheme because this scheme is often used in high-speed quadcopters and is easy to control.

Since one of the basic requirements for the quadcopters is the minimum mass, and the other requirement in this article is the deflected thrust vector, it is preferable to use fewer servos, while simultaneously tilting all axes of the propeller group. Structurally, this synchronous tilting of the axes can be achieved using two servo drives and a parallel transfer mechanism. The parallel transfer mechanism, which deflects all axes by one angle, leaving them parallel to each other, consists of the main structural elements:

- Identical crosses (eight in total)

- Hooke's joints (eight in total)

- Scotch yokes (two in total, crossed) united on plate with slots

- Servos (two in total)

Two servos through levers fix the mechanism in the required angular position of the axes relative to the body. 
A general view of the proposed construction of a quadrocopter in different angles, scales, and details for a better understanding is presented in several figures at once (see Fig. 2-7).
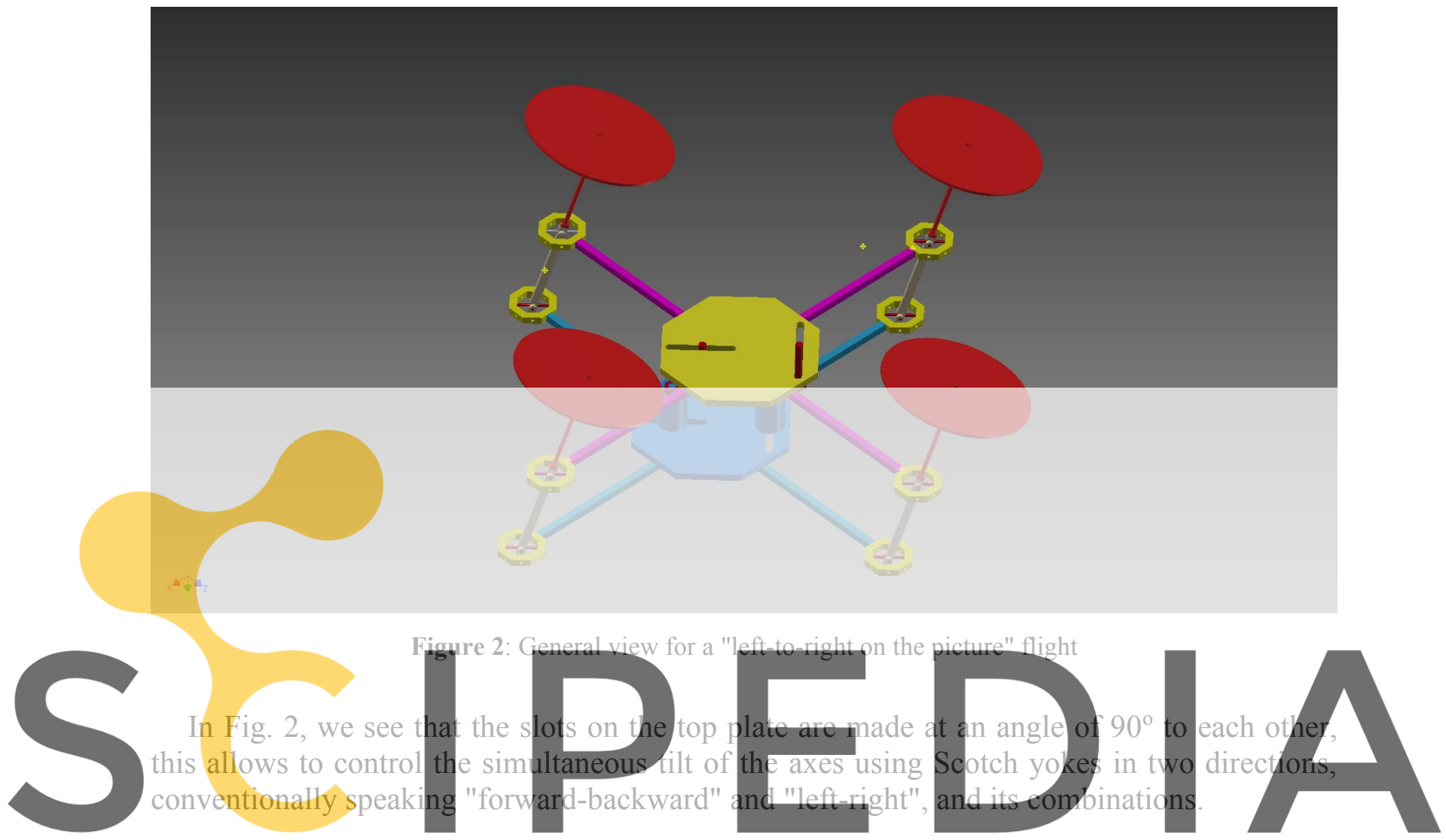

Register for free at https//www.scipedia.com to download the version without the watermark

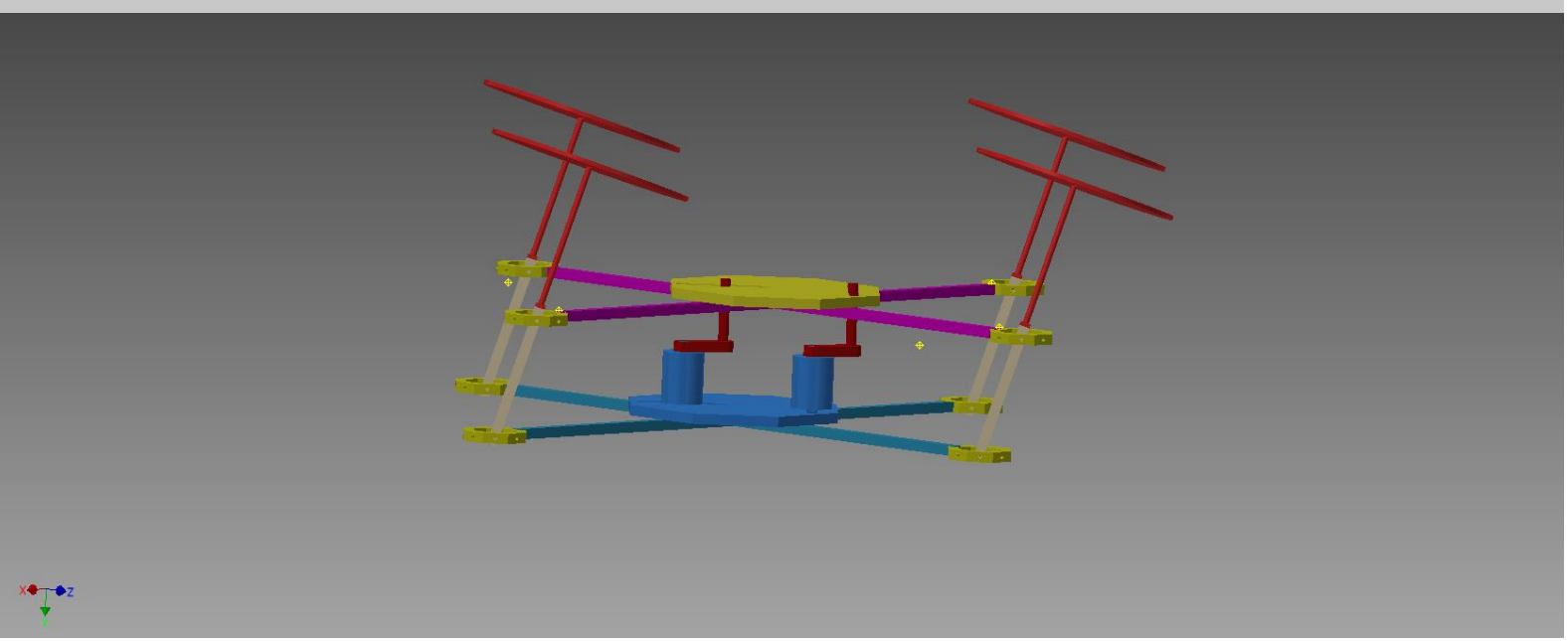

Figure 3: Side view for "left-to-right on the picture" flight 
In Fig. 3 you can see how the arms of the servos are rotated, at the ends of which there are rods inserted into the slots of the top plate.
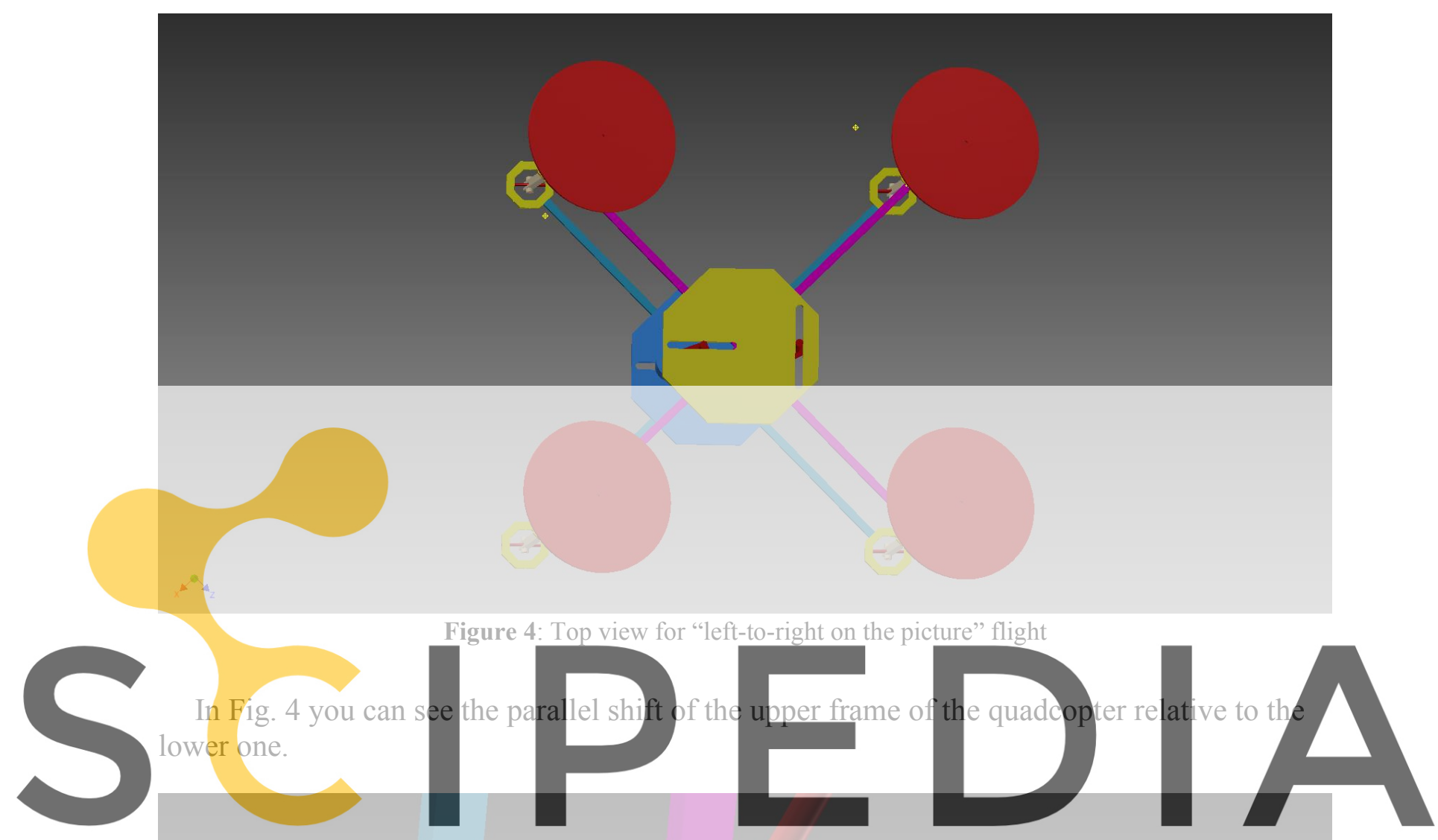

Register for free at https//www.scipedia.com to download the version without the watermark

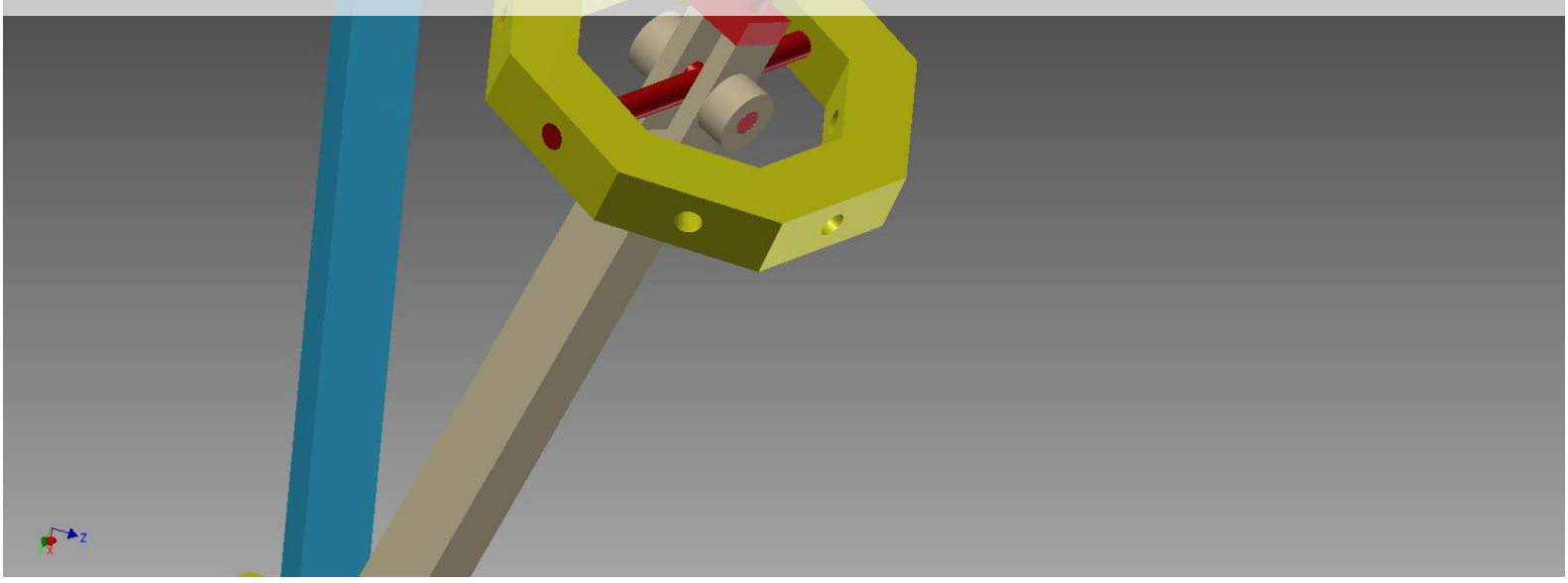

Figure 5: Hooke's joint in the connection of the axis with the body 
In Fig. 5 you can see that the cross inside the Hooke's joint is attached to the quadcopter beam on one side, and to the quadcopter axis mount on the other side.
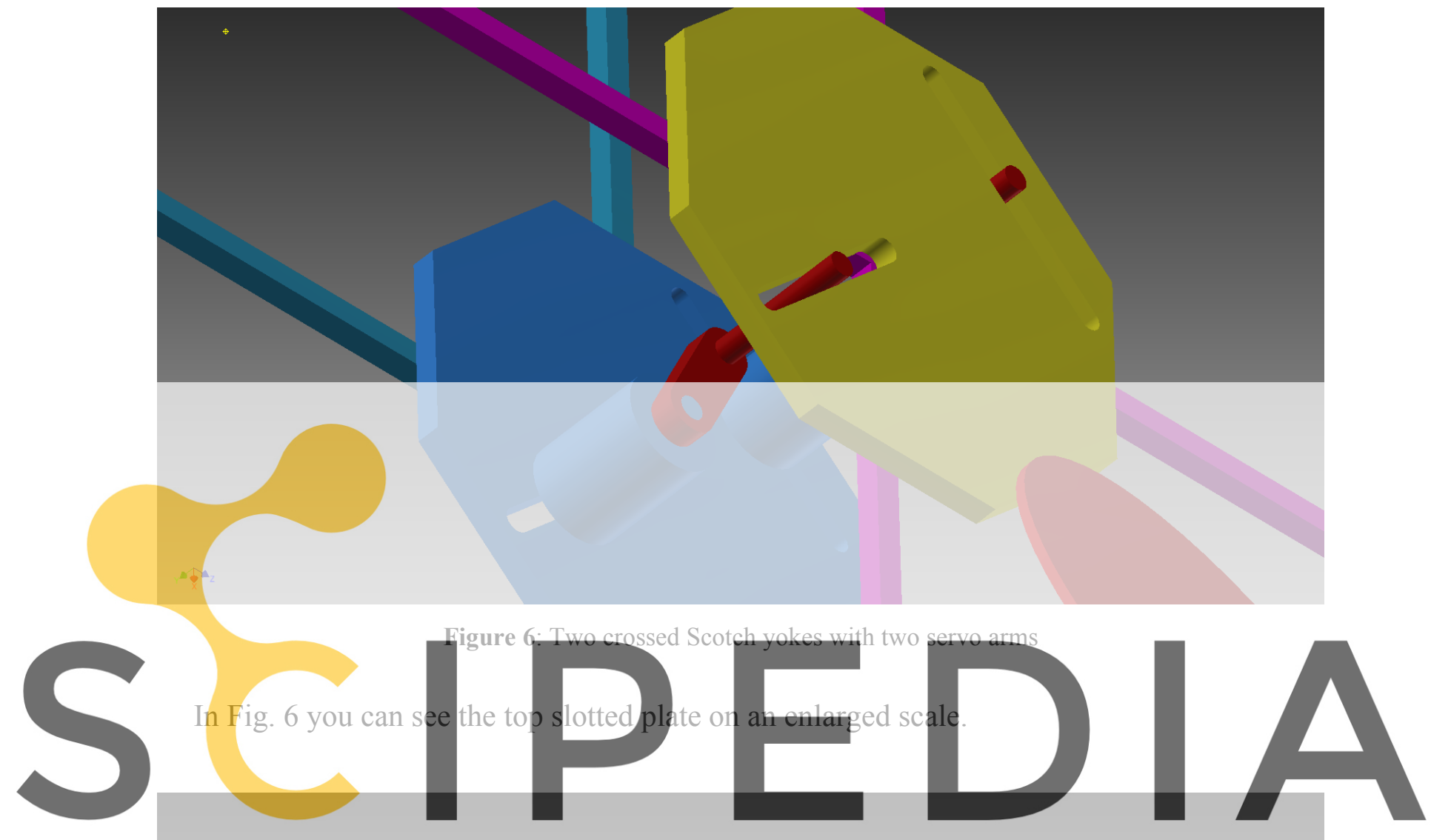

Register for free at https//www.scipedia.com to download the version without the watermark

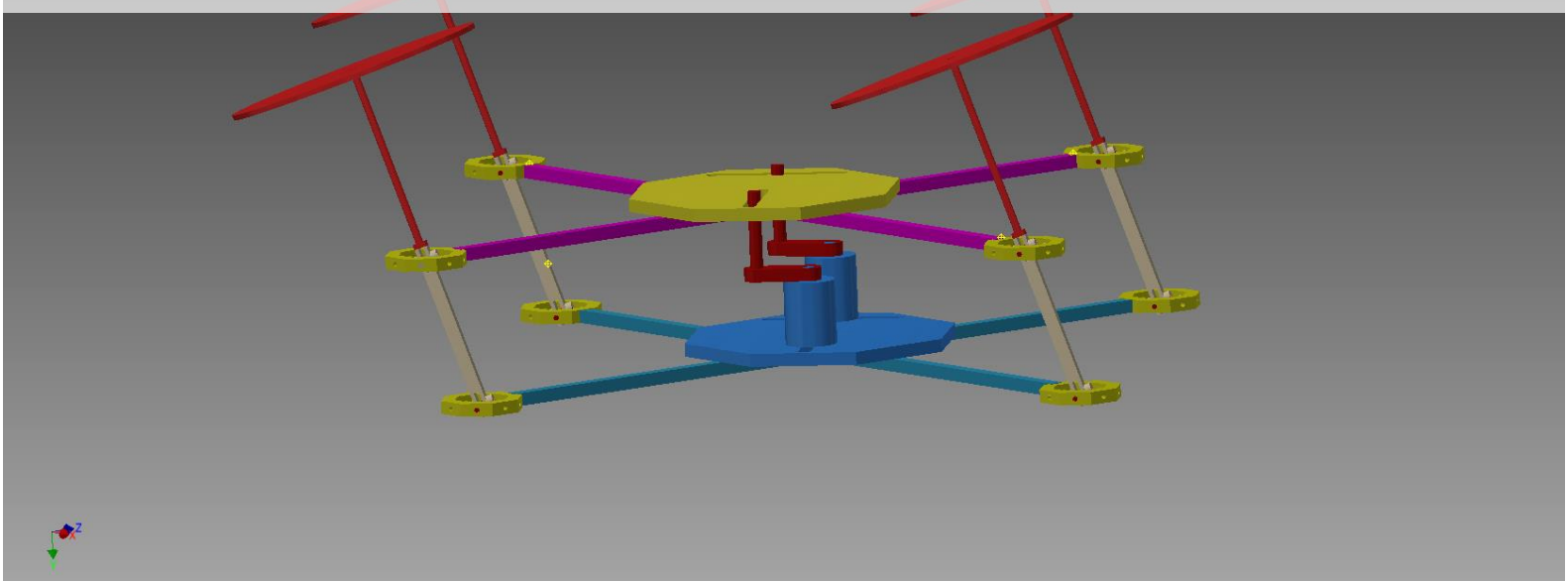

Figure 7: A general view for a "right-to-left on the picture" flight with a different position of the servos while keeping the roll, pitch, and yaw angles of the quadcopter the same as in previous figures 
In Fig. 7 we can see that we can change the direction of flight by setting a different position to the servos, and at the same time, the roll, pitch, and yaw angles of the quadcopter will not change.

Note that the motors that drive the propellers are not shown in the illustrations. This is because there are various options for their placement on tilted axles (under the lower platform, between platforms, above the upper platform).

Also, in this article, the proportions of the construction are not considered in detail, but only the basic concept of a quadcopter with a thrust vector control based on Scotch yoke is proposed. At this conceptual stage, we also do not pay attention to design constraints, such as the maximum possible angles of inclination of the propeller axles, reliability and durability of the mechanism, and so on.

\section{GENERAL DYNAMICS}

A mathematical model of the dynamics of a quadcopter can be considered using Newton's laws and dynamic Euler equations in the reference frame associated with the body of the quadcopter, where the designation with the subscript "B" means "Body frame" [10]:

$$
\begin{gathered}
m\left(\vec{\omega}_{B} \times \vec{V}_{B}+\dot{\vec{V}}_{B}\right)=\vec{f}_{B}, \\
\hat{I} \dot{\vec{\omega}}_{B}+\vec{\omega}_{B} \times\left(\hat{I} \vec{\omega}_{B}\right)=\vec{m}_{B},
\end{gathered}
$$

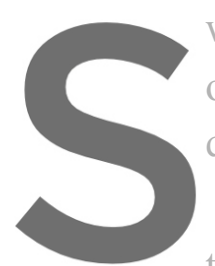

where $m$-quadrotor mass, $\vec{\omega}$ - angutar velocity of quadi

of quadrotor, $\vec{I}-\operatorname{diag}$

quadrotor, $\vec{m}_{B}-$ sum

Then angular velocity

the inertial reference

local region [10]:
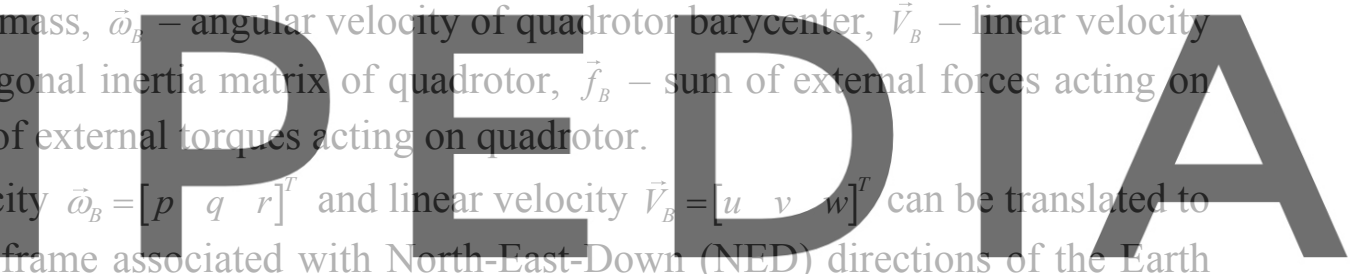

where $\vec{V}=\left[\begin{array}{lll}\dot{x} & \dot{y} & \dot{z}\end{array}\right]^{T}$ - linear velocity in NED frame, $\vec{\omega}=\left[\begin{array}{ccc}\dot{\phi} & \dot{\theta} & \dot{\psi}\end{array}\right]^{T}$ - angular velocity in in roll-pitch-yaw Euler angles, $R$ - rotation matrix based on roll-pitch-yaw Euler angles, $T-$ matrix of angular transformations [10]:

$$
\begin{aligned}
R & =\left[\begin{array}{ccc}
c_{\theta} c_{\psi} & s_{\phi} s_{\theta} c_{\psi}-c_{\phi} s_{\psi} & c_{\phi} s_{\theta} c_{\psi}+s_{\phi} s_{\psi} \\
c_{\theta} s_{\psi} & s_{\phi} s_{\theta} s_{\psi}+c_{\phi} c_{\psi} & c_{\phi} s_{\theta} s_{\psi}-s_{\phi} c_{\psi} \\
-s_{\theta} & s_{\phi} c_{\theta} & c_{\phi} c_{\theta}
\end{array}\right], \\
T & =\left[\begin{array}{ccc}
1 & s_{\phi} t_{\theta} & c_{\phi} t_{\theta} \\
0 & c_{\phi} & -s_{\phi} \\
0 & s_{\phi} / c_{\theta} & c_{\phi} / c_{\theta}
\end{array}\right],
\end{aligned}
$$

where $c_{*}=\cos (*), s_{*}=\sin (*), t_{*}=\tan (*)$.

As for forces and torques we can write down the following: 


$$
\begin{aligned}
& \vec{f}_{B}=m g R^{T} \vec{e}_{z}+\vec{f}_{t}+\vec{f}_{w}, \\
& \vec{m}_{B}=\vec{\tau}_{t}+\vec{\tau}_{g}-\vec{g}_{a}+\vec{\tau}_{w},
\end{aligned}
$$

where $\vec{e}_{z}$ - the unit vector in the inertial frame directed "down" (to the Earth center), $\vec{f}_{t}-$ total thrust vector generated by all propellers, $\vec{f}_{w}=\left[\begin{array}{lll}f_{w x} & f_{w y} & f_{w z}\end{array}\right]^{T}-$ aerodynamic force produced by oncoming wind on the quadrotor, $\vec{\tau}_{t}=\left[\begin{array}{lll}\tau_{x} & \tau_{y} & \tau_{z}\end{array}\right]^{T}-$ torque, generated by total thrust from four propellers with different angular velocities, $\vec{\tau}_{g}$ - torque in the gravity field that appears when propeller axles incline (it is in our construction), $\vec{g}_{a}-$ gyroscopic moment caused by rotation of four propellers and quadrotor body, $\vec{\tau}_{w}=\left[\begin{array}{lll}\tau_{w x} & \tau_{w y} & \tau_{w z}\end{array}\right]^{T}-$ aerodynamic torque, produced by oncoming wind on the quadrotor.

In what follows, we will number the propellers as follows (see Fig. 8).
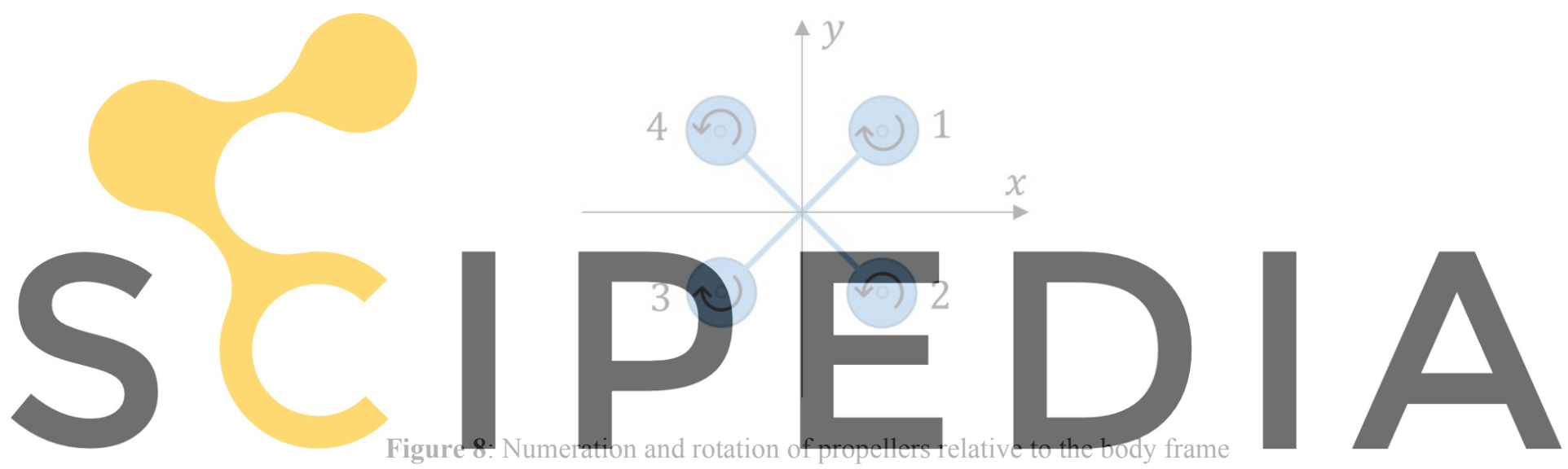

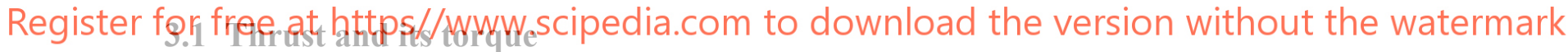

In a conventional quadcopter, the total thrust vector (and thrust of each propeller) is always directed along the $z$-axis.

In our work, we consider the thrust of each propeller at an angle to the $z$ axis, and to the $x$ and $y$ axes of the body frame. Assuming that the thrust of each propeller is approximately proportional to the square of its rotation speed [11], we write down the total thrust (see Fig. 9):

$$
\vec{f}_{t}=\left[\begin{array}{lll}
f_{t x} & f_{t y} & f_{t z}
\end{array}\right]^{T}=\sum_{i=1}^{4} \vec{f}_{i}=b \sum_{i=1}^{4} \Omega_{i}^{2}\left[s\left(\alpha_{i}\right) c\left(\beta_{i}\right) \quad s\left(\alpha_{i}\right) s\left(\beta_{i}\right)-c\left(\alpha_{i}\right)\right]^{T},
$$

where $b$ - thrust coefficient, $\vec{f}_{i}-$ thrust of $i$-propeller, $\Omega_{i}-$ angular speed of the $i$ propeller, $\alpha_{i}$ - angle between the $i$-propeller axle and $z$ axis, $\beta_{i}$ - angle between the projection of $i$-propeller axle on $x y$-plane and $x$ axis. 


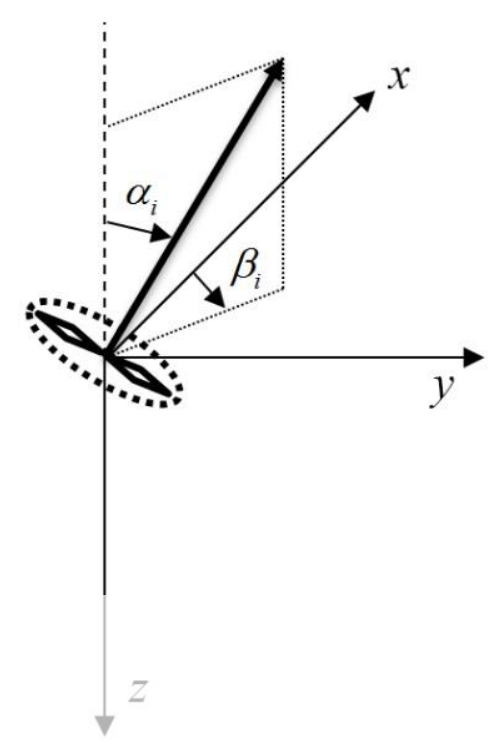

Figure 9: Orientation angles of propeller axle in the body frame

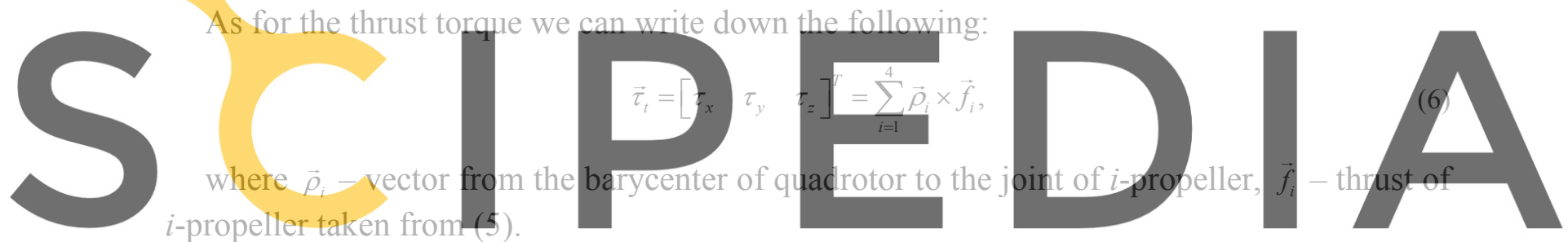

The vector $\vec{\rho}_{i}$ depends on the geometry of the quadrotor. Let us assume that on Fig. 8 we

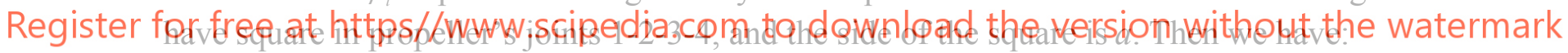

$$
\begin{array}{ll}
\vec{\rho}_{1}=\left[\begin{array}{lll}
a / \sqrt{2} & a / \sqrt{2} & 0
\end{array}\right]^{T}, & \vec{\rho}_{2}=\left[\begin{array}{lll}
a / \sqrt{2} & -a / \sqrt{2} & 0
\end{array}\right]^{T}, \\
\vec{\rho}_{3}=\left[\begin{array}{lll}
-a / \sqrt{2} & -a / \sqrt{2} & 0
\end{array}\right]^{T}, & \vec{\rho}_{4}=\left[\begin{array}{lll}
-a / \sqrt{2} & a / \sqrt{2} & 0
\end{array}\right]^{T} .
\end{array}
$$

Then, considering (5)-(7) and the fact that in our construction all propeller axles are inclined the same angles, we obtain:

$$
\begin{aligned}
& \alpha_{1}=\alpha_{2}=\alpha_{3}=\alpha_{4}=\alpha \in\left[-\alpha^{\max }, \alpha^{\max }\right], \quad \beta_{1}=\beta_{2}=\beta_{3}=\beta_{4}=\beta \in[-\pi, \pi], \\
& \vec{f}_{t}=b\left(\Omega_{1}^{2}+\Omega_{2}^{2}+\Omega_{3}^{2}+\Omega_{4}^{2}\right)[s(\alpha) c(\beta) s(\alpha) s(\beta)-c(\alpha)]^{T}, \\
& \tau_{x}=\frac{a b}{\sqrt{2}}\left(-\Omega_{1}^{2}+\Omega_{2}^{2}+\Omega_{3}^{2}-\Omega_{4}^{2}\right) c(\alpha), \\
& \tau_{y}=\frac{a b}{\sqrt{2}}\left(\Omega_{1}^{2}+\Omega_{2}^{2}-\Omega_{3}^{2}-\Omega_{4}^{2}\right) c(\alpha), \\
& \tau_{z}=\frac{a b}{\sqrt{2}}\left(\left(-\Omega_{1}^{2}+\Omega_{2}^{2}+\Omega_{3}^{2}-\Omega_{4}^{2}\right) c(\beta)+\left(\Omega_{1}^{2}+\Omega_{2}^{2}-\Omega_{3}^{2}-\Omega_{4}^{2}\right) s(\beta)\right) .
\end{aligned}
$$




\subsection{Gravity torque}

Torque in the gravity field generated by axles inclination can be determined by this expression:

$$
\vec{\tau}_{g}=\sum_{i=1}^{4} \vec{\sigma}_{i} \times \mu \vec{g}
$$

where $\vec{\sigma}_{i}$ - vector from the barycenter of fixed part of quadrotor to the barycenter of $i$-th propeller-motor group, $\mu \vec{g}$ - gravity force, acting on $i$-th propeller-motor group.

The value of $\vec{\tau}_{g}$ characterizes the disbalance of total barycenter of quadrotor, and because of the masses shift it creates incline torque. By the way, this also changes the inertia matrix of quadrotor. However, if masses and its shift are not so big, we can neglect it. For this, it is necessary that the barycenter of each propeller-motor group should be fixed in the body frame when the propeller axles are inclined.

\subsection{Gyroscopic torque}

\section{Gyroscopic torque can be determined as follows:}

$$
\vec{g}_{a}=\sum_{i=1}^{4} J_{p}\left(\vec{\omega}_{B} \times \vec{e}_{i}\right)(-1)^{i+1} \Omega_{i},
$$

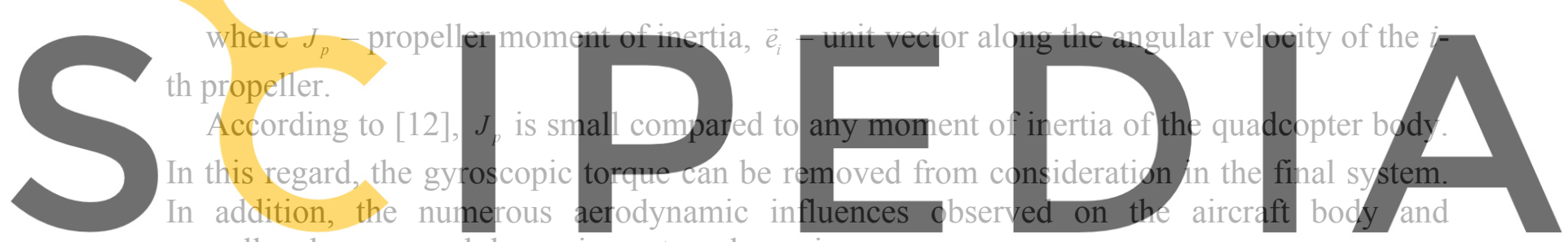

propellers have a much larger impact on dynamics.

\section{Register for free at httips/Amww.scipedia.com to download the version without the watermark}

Aerodynamic effects can be divided into body-related effects and propeller-related effects.

Body-related effects mainly consist of drag force, lift force, and lateral force. All these forces can be described by approximate expressions:

$$
f_{D}=c_{D} \frac{\rho v^{2}}{2} S, f_{L}=c_{L} \frac{\rho v^{2}}{2} S, f_{Y}=c_{Y} \frac{\rho v^{2}}{2} S,
$$

where $\rho v^{2} / 2$ - air flow pressure, $\rho$-air density, $v$ - air flow velocity, $c_{D}$ - drag coefficient, $c_{L}$ - lift coefficient, $c_{Y}$ - lateral force coefficient, $S$ - area of the largest cross-section of the quadcopter body, perpendicular to the incoming air flow.

In our quadcopter design, due to which it is possible to fly at a zero pitch angle (as well as at pitch angles of different signs), firstly, it is possible to reduce the cross-sectional area, and therefore to reduce the drag force, and secondly, to create the effect of a lifting wing, thanks to which to compensate for the effect of gravity, and direct the thrust to move forward, and thereby increase the speed and duration of the flight.

As for propeller-related aerodynamic effects, many of them are described [11], but most of 
all we will pay attention to the drug torque, which allows the quadrotor to change yaw-angle. The $i$-th propeller-drag torque can be approximately described by:

$$
\vec{\tau}_{D(i)}=-\operatorname{sgn}\left(\Omega_{i}\right) k \Omega_{i}^{2} \vec{e}_{i},
$$

where $k$-constant, $\vec{e}_{i}$ - unit vector along the direction of the propeller axle.

The total propeller-drag torque will be:

$$
\vec{\tau}_{D}=k\left(\Omega_{1}^{2}-\Omega_{2}^{2}+\Omega_{3}^{2}-\Omega_{4}^{2}\right)[s(\alpha) c(\beta) s(\alpha) s(\beta)-c(\alpha)]^{T} .
$$

All these aerodynamic effects must be included in $\vec{f}_{w}$ and $\vec{\tau}_{w}$ in (4).

\section{CONCLUSIONS}

- $\quad$ Newest Scotch yoke thrust vectoring quadcopter design introduced.

- The design allows the quadcopter to fly with zero pitch and roll angles.

The absence of an inclination of the quadrocopter body reduces the characteristic cross-section of the aircraft relative to the incoming air flow. This reduces the aerodynamic drag of the quadcopter. As a consequence, this leads to an increase in flight speed and a decrease in power consumption.

If we make the body in the form of a wing, so that there is a lift force, then it can be achieved that it will compensate for the force of gravity and the entire thrust of the

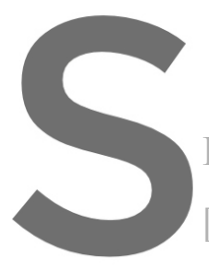
quadcopter can be directed towards f
to provide the "Cruise" mode for high-
RENCES
1, M., Bülthoff, H.H. and Giordano, P
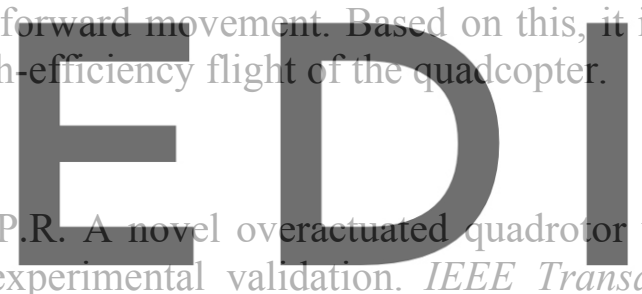

aerial vehicle: modeling, control, and experimental validation. IEEE Transactions on Control Systems Technology (2015) 23(2):540-556 (DOI:10.1109/TCST.2014.2330999),

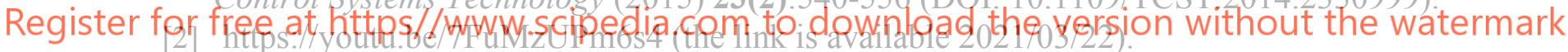

[3] Cutler, M. Design and control of an autonomous variable-pitch quadrotor helicopter. Ph.D. dissertation, Citeseer (2012).

[4] Cutler, M. and How, J.P. Actuator constrained trajectory generation and control for variable-pitch quadrotors. Proceeding of the AIAA Guidance, Navigation, and Control Conference (GNC), Minneapolis, MN, USA (2012).

[5] Fresk, E. Modeling, control and experimentation of a variable pitch quadrotor. Master's Thesis, Luleå University of Technology, Luleå, Sweden (2013).

[6] Hua, M.-D., Hamel, T., and Samson, C. Control of VTOL vehicles with thrust-direction tilting, Proc. of the 19th IFAC World Congress (2014).

[7] Nemati, A., and Kumar, M. Modeling and control of a single axis tilting quadcopter. 2014 American Control Conference, pp. 3077-3082 (2014).

[8] Riccardi, F., Panizza, P., Lovera, M. Identification of the attitude dynamics for a variablepitch quadrotor UAV. Proceeding of the European Rotorcraft Forum (ERF) 2014, Southampton, UK (2014).

[9] Sheng, S. and Sun, C. Control and optimization of a variable-pitch quadrotor with minimum power consumption. Energies (2016) 9(4): 232. 
[10] Sabatino, F. Quadrotor control: modeling, nonlinear control design, and simulation. Master's degree project. Stockholm, Sweden (2015).

[11] Bristeau, P.J., Martin, P., Salaun, E. and Petit, N.. The role of propeller aerodynamics in the model of a quadrotor UAV. Proceedings of the European Control Conference (2009) 683-688.

[12] Mellinger, D., Lindsey, Q., Shomin, M. and Kumar, V. Design, modeling, estimation and control for aerial grasping and manipulation. IEEE/RSJ International Conference on Intelligent Robots and Systems (IROS) (2011) 2668 -2673. 